\title{
Glass transition and relaxation dynamics of propylene glycol-water solutions confined in clay
}

\author{
Khalid Elamin, Jimmy Björklund, Fredrik Nyhlén, Madeleine Yttergren, Lena Mårtensson, \\ and Jan Swenson ${ }^{\mathrm{a}}$ \\ Department of Applied and Physics, Chalmers University of Technology, SE-412 96 Göteborg, Sweden
}

(Received 14 March 2014; accepted 27 June 2014; published online 17 July 2014)

\begin{abstract}
The molecular dynamics of aqueous solutions of propylene glycol (PG) and propylene glycol methylether (PGME) confined in a two-dimensional layer-structured Na-vermiculite clay has been studied by broadband dielectric spectroscopy and differential scanning calorimetry. As typical for liquids in confined geometries the intensity of the cooperative $\alpha$-relaxation becomes considerably more suppressed than the more local $\beta$-like relaxation processes. In fact, at high water contents the calorimetric glass transition and related structural $\alpha$-relaxation cannot even be observed, due to the confinement. Thus, the intensity of the viscosity related $\alpha$-relaxation is dramatically reduced, but its time scale as well as the related glass transition temperature $T_{g}$ are for both systems only weakly influenced by the confinement. In the case of the PGME-water solutions it is an important finding since in the corresponding bulk system a pronounced non-monotonic concentration dependence of the glass transition related dynamics has been observed due to the growth of hydrogen bonded relaxing entities of water bridging between PGME molecules [J. Sjöström, J. Mattsson, R. Bergman, and J. Swenson, Phys. Chem. B 115, 10013 (2011)]. The present results suggest that the same type of structural entities are formed in the quasi-two-dimensional space between the clay platelets. It is also observed that the main water relaxation cannot be distinguished from the $\beta$-relaxation of PG or PGME in the concentration range up to intermediate water contents. This suggests that these two processes are coupled and that the water molecules affect the time scale of the $\beta$-relaxation. However, this is most likely true also for the corresponding bulk solutions, which exhibit similar time scales of this combined relaxation process below $T_{g}$. Finally, it is found that at higher water contents the water relaxation does not merge with, or follow, the $\alpha$-relaxation above $T_{g}$, but instead crosses the $\alpha$-relaxation, indicating that the two relaxation processes are independent of each other. This can only occur if the two processes do not occur in the same parts of the confined solutions. Most likely the hydration shell of the interlayer $\mathrm{Na}^{+}$ions is causing this water relaxation, which does not participate in the $\alpha$-relaxation at any temperature. (C) 2014 AIP Publishing LLC. [http://dx.doi.org/10.1063/1.4889742]
\end{abstract}

\section{INTRODUCTION}

Propylene glycol (PG) and propylene glycol methylether (PGME) are typical glass-forming molecular liquids, commonly used for studies of glass transition related dynamics of supercooled liquids. These liquids have also been successfully used as model systems of aqueous solutions and hydrogen bonded liquids due to their completely different behaviors when they are mixed with water. PG has two $\mathrm{OH}$ end groups and therefore these molecules can form chains of hydrogen bonded molecules, whereas PGME has only one $\mathrm{OH}$ end group, giving the ability to hydrogen bond to only one other PGME molecule. Thus, PGME can only form pairs of two molecules, and this difference in the ability to form hydrogen bonds with other molecules is clearly shown up in the different dynamical properties of PG and PGME. For example, the glass transition temperature $\left(T_{g}\right)$ of $\mathrm{PG}$ is about $168 \mathrm{~K}$, to compare with the $143 \mathrm{~K}$ for PGME. Furthermore, when the

\footnotetext{
a) Author to whom correspondence should be addressed. Electronic mail: jan.swenson@chalmers.se
}

density of hydrogen bonds increases by adding water the two systems behave completely different. Whereas $T_{g}$ decreases slightly with increasing water content (up to a water concentration of about $60 \mathrm{wt}$. \% where partial crystallization occurs) for PG, a completely different behavior is observed for PGME. ${ }^{1}$ In the latter case $T_{g}$ increases rapidly with increasing water content up to a concentration of $55 \mathrm{wt} \%$ water, where $T_{g}$ is as high as $169 \mathrm{~K}$, before it decreases with even more water added. ${ }^{1}$ Therefore, despite the fact that $T_{g}$ of pure PG is $25 \mathrm{~K}$ higher than for pure PGME, $T_{g}$ of a $50 \mathrm{wt}$. \% aqueous solution of PGME is higher than for the corresponding solution of PG. ${ }^{1}$ These completely different influences of water on the dynamical properties can be explained by the possibility that water does not significantly alter the chain-like structure of hydrogen bonded PG molecules, and therefore added water acts as a plasticizer in a similar way as for polymers. For PGME, on the other hand, results have indicated that water has the tendency to bridge between two or more PGME molecules, and thereby forming larger structural entities than single pairs of two PGME molecules. ${ }^{2}$ These larger structural entities should relax slower and therefore giving rise to an 
increase of $T_{g}$. However, also these entities should reach a finite size maximum, and therefore it is likely that this limit is reached at about $55 \mathrm{wt}$. \% water where $T_{g}$ starts to decrease. ${ }^{2}$

This example of bulk aqueous solutions shows the strong influence of hydrogen bonds on the dynamics of glassforming liquids. It is clear that considerable knowledge about the role of hydrogen bonds can be gained. This knowledge can be further extended by imposing geometrical constraints, which may affect the ability of the molecules to interact via hydrogen bonds. This fact, in addition to the great biological, geological, and technological interest in geometrically confined water and aqueous solutions has caused a rapidly growing interest of such confined systems. ${ }^{3-17}$ Issues of importance to explore are, for instance, how the hydrogen bonds in the corresponding bulk system are altered in different types of confined geometries, and how this, in turn, affects the glass transition related dynamics. In this paper we have used broadband dielectric spectroscopy and differential scanning calorimetry (DSC) to explore how the dynamical bulk behaviors of aqueous solutions of PG and PGME are altered by the intercalation in a two-dimensional vermiculite clay. From the results it is evident that the dynamics of both solutions is only slightly affected by the severe geometrical constraint in one direction, suggesting that the amount and nature of the hydrogen bonds are basically preserved in the quasi-twodimensional space between the clay platelets, particularly at low and intermediate water contents.

\section{EXPERIMENTAL}

Propylene glycol (PG) (HO-CH $\left.\left(\mathrm{CH}_{3}\right)-\mathrm{CH}_{2}-\mathrm{OH}\right)$ and propylene glycol methylether (PGME) $\left(\mathrm{CH}_{3}-\mathrm{O}-\right.$ $\left.\mathrm{CH}\left(\mathrm{CH}_{3}\right) \mathrm{CH}_{2}-\mathrm{OH}\right)$ were purchased from Sigma-Aldrich and freeze-dried before measurements. ${ }^{18}$ These liquids were mixed with distilled milli-Q water (conductivity $<0.1 \mu \mathrm{S} / \mathrm{m}$ ) to obtain aqueous solutions in the whole concentration range from 0 to $100 \mathrm{wt}$. \% water. To ensure complete mixing the solutions were put into an ultrasonic bath for $15 \mathrm{~min}$.

As confining host material, a vermiculite clay provided by Askania, Sweden, was used. The clay pieces were washed and then treated for more than one year with $1 \mathrm{M} \mathrm{NaCl}$ solution at room temperature, with regular change of the solution, to produce a pure Na-vermiculite. A totally dry Na-vermiculite clay was obtained by drying at $120^{\circ} \mathrm{C}$ in a vacuum oven for $48 \mathrm{~h}$. After the drying the clay pieces were submerged in the different solutions and stored in bottles at $50^{\circ} \mathrm{C}$ for at least one week to ensure full intercalation of the solutions. Immediately before measurements the clay pieces were dried from bulk solutions with tissues.

$\mathrm{X}$-ray diffraction measurements were performed on the clay samples containing the single liquids of PG, PGME, and water to determine the spacing between two consecutive clay layers ( $d$-spacing) and to ensure that most layers were filled with liquids. The results showed that the $d$-spacing was relatively well-defined for all liquids, but clearly different for the three samples. For PGME the value was approximately $12.5 \AA$, for PG $14.1 \AA$ and for water $15.0 \AA$, giving interlayer spacing of about $3.9 \AA$, $5.5 \AA$, and $6.4 \AA$, respectively, with the thickness of the actual clay platelet subtracted. However, although basically all layers were filled with liquids, weigh measurements showed that the average densities of the intercalated liquids were substantially lower (about 30\%) than the corresponding bulk liquids. Therefore, the X-ray diffraction and weight measurements indicate that the solutions are somewhat heterogeneously distributed and only partly fill the interlayer spacing.

\section{A. DSC measurements}

DSC measurements were performed on the TA Instruments DSC Q1000. Samples (5-20 mg) of the intercalated solutions were placed in hermetically sealed aluminum pans immediately before the DSC measurements were performed. The glass transition temperature $\left(T_{g}\right)$ of each sample was measured at a heating rate of $10 \mathrm{Kmin}^{-1}$ after been cooled rapidly from $313 \mathrm{~K}$ to $123 \mathrm{~K}$ at a rate of $-30 \mathrm{Kmin}^{-1}$. The $T_{g}$ value was determined as the inflection point of the step in heat capacity. Three samples of each concentration were measured in order to ensure repeatability and to determine the sampleto-sample variability, which was used to estimate the experimental uncertainty of the measured $T_{g}$-values.

\section{B. Dielectric relaxation measurements}

Dielectric relaxation measurements were performed on a broadband dielectric spectrometer from Novocontrol, equipped with a Novocontrol Alfa-S High Resolution Dielectric Analyzer. Each sample consisted of a few clay pieces with a similar thickness of typically $0.3-0.5 \mathrm{~mm}$. These pieces were placed between two gold-plated brass electrodes of diameter $20 \mathrm{~mm}$. Measurements were performed in the frequency and temperature ranges $10^{-2}-10^{6} \mathrm{~Hz}$ and $120-300 \mathrm{~K}$, respectively. Isothermal (within $\pm 0.02 \mathrm{~K}$ ) frequency scans of the complex dielectric function, $\varepsilon^{*}(f)=\varepsilon^{\prime}(f)-i \varepsilon^{\prime \prime}(f)$, were performed every fifth degree.

\section{RESULTS AND DISCUSSION}

The insets of Figs. 1(a) and 1(b) show DSC heating scans for PG-water and PGME-water, respectively, confined in Navermiculite clay. A calorimetric glass transition could be clearly observed only in the concentration range $0-60 \mathrm{wt}$. \% water. The reason for why a clear $T_{g}$ cannot be observed at high water contents is that confined water does not exhibit any evident $T_{g}$ by ordinary DSC, as further discussed in Ref. 3. In Fig. 1 the obtained $T_{g}$-values are shown for the intercalated PG-water (a) and PGME-water (b) mixtures in comparison with the corresponding $T_{g}$ of the bulk solutions. ${ }^{1}$ It can be seen that for the intercalated PG-water solutions $T_{g}$ is the same, within the experimental errors, as for the corresponding bulk solutions, and it changes only a few $\mathrm{K}$ in the shown concentration range. For the PGME-water solutions the concentration dependence is, as discussed above, very different and a strong increase of $T_{g}$ with about $25 \mathrm{~K}$ is observed in the concentration range up to $50 \mathrm{wt}$ \% water. However, also for this system the concentration behavior is very similar for the intercalated and bulk solutions, 

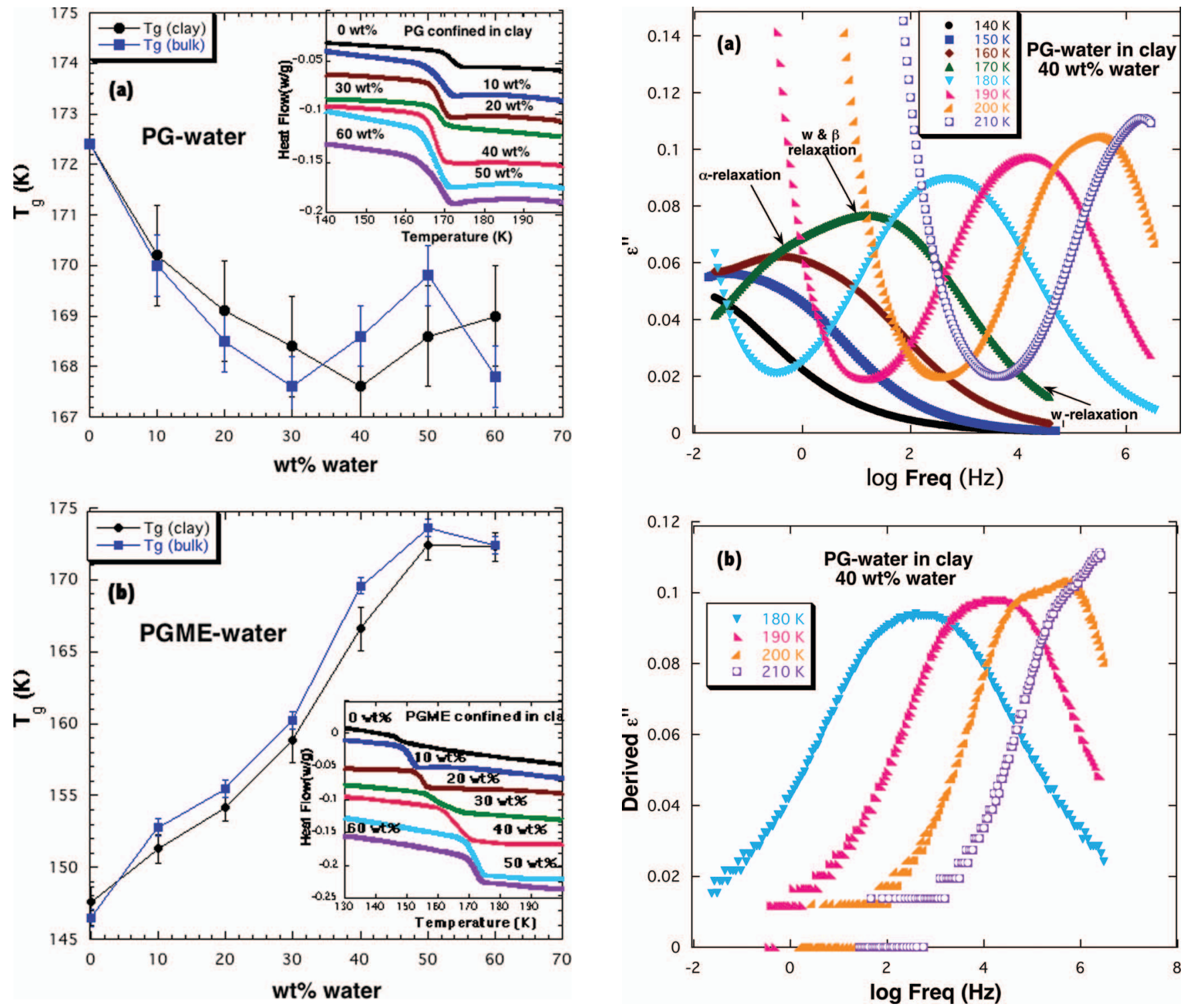

FIG. 1. Concentration dependences of the calorimetric glass transition temperature, $T_{g}$, of (a) PG-water mixtures and (b) PGME-water mixtures confined in a Na-vermiculite clay. $T_{g}$-values of the corresponding bulk solutions ${ }^{1}$ are also shown for comparison. The insets show the DSC heating scans from which the $T_{g}$-values were determined as the inflection point in the step of the heat flow (in contrast to the case in Ref. 1 where the onset values of $T_{g}$ were shown). The curves have been vertically shifted for clarity.

indicating that basically the same growth of size of relaxing structural entities occurs in the confined geometry. Hence, this suggests that water molecules are able to form hydrogen bonded bridges between different PGME molecules also in the present quasi-two-dimensional geometry, i.e., the clusters of water and PGME molecules are able to adapt their shape to the two-dimensional geometry.

Fig. 2 shows the imaginary part of the dielectric permittivity as a function of frequency for an intercalated PG-water solution containing $40 \mathrm{wt}$. \% water, in the temperature range $130-200 \mathrm{~K}$. In the low temperature range a clearly asymmetric dielectric loss peak is observed, containing contributions from two water relaxations, and most likely also from the $\beta$-relaxation of PG, as further discussed below. At about $170 \mathrm{~K}$ the glass transition related structural $\alpha$-relaxation of

FIG. 2. Measured (a) and derived values, using Eq. (1), (b) of the imaginary part of the dielectric permittivity of the intercalated PG-water mixture containing $40 \mathrm{wt}$. \% water, at the selected temperatures given in the figures. The observed relaxation processes are also indicated in Figure 2(a).

the solution enters the low frequency range as a shoulder. This $\alpha$-relaxation moves closer to the main water $(w)$ relaxation and the $\beta$-relaxation of PG with increasing temperature, due to its stronger temperature dependence, and above $180 \mathrm{~K}$ it is difficult to distinguish the different relaxation processes. To ensure that no additional relaxation processes are present, e.g., hidden in the strong conductivity contribution at low frequencies and high temperatures, we also derived the imaginary part of the dielectric permittivity from its real part. If we assume that possible additional relaxation processes are not substantially broader than a Debye peak the following relation between the imaginary and real parts of the permittivity is valid: ${ }^{19}$

$$
\varepsilon^{\prime \prime}(\omega)=\sqrt{-\frac{\Delta \varepsilon}{2}} \frac{d \varepsilon^{\prime}(\omega)}{d \ln (\omega)}
$$


where $\omega$ is the angular frequency and $\Delta \varepsilon=\varepsilon_{s}-\varepsilon_{\infty}$ is the difference in the limiting values of the permittivity at low and high frequencies. The advantage of this derived value of the imaginary part of the dielectric permittivity is that it does not contain any conductivity contribution, in contrast to the measured data presented in Fig. 2(a). This is evident in Fig. 2(b), where values derived from Eq. (1) are shown for some selected temperatures. From this figure it is also clear that no additional relaxation processes become visible, e.g., in the low frequency range where the conductivity contribution dominates in Fig. 2(a). Therefore, only up to four standard fit functions were needed to describe the spectra in Fig. 2(a); a power law for the dc-conductivity, a HavriliakNegami function ${ }^{20}$ for the $\alpha$-relaxation and Cole-Cole functions ${ }^{21}$ for the $w$-relaxation (or $\beta$-relaxation) and the more local water relaxation $\left(w_{l}\right)$. Hence, the imaginary part of the measured permittivity was described by

$$
\begin{aligned}
\varepsilon^{\prime \prime}(\omega)= & \frac{\sigma}{i \varepsilon_{0} \omega^{n}}+\frac{\Delta \varepsilon_{\alpha}}{\left(1+\left(i \omega \tau_{\alpha}\right)^{a}\right)^{b}}+\frac{\Delta \varepsilon_{w}}{1+\left(i \omega \tau_{w}\right)^{a}} \\
& +\frac{\Delta \varepsilon_{w l}}{1+\left(i \omega \tau_{w l}\right)^{a}},
\end{aligned}
$$

where $\sigma$ is the dc conductivity, $\tau_{\alpha}, \tau_{w}$, and $\tau_{w l}$ are the relaxation times for the $\alpha, w$ (with likely contribution from the $\beta$ relaxation of PG) and $w_{l}$ relaxations, respectively, $\Delta \varepsilon_{\alpha}, \Delta \varepsilon_{w}$, and $\Delta \varepsilon_{w l}$ are the dielectric strengths of the same processes, and $a$ and $b$ are the shape parameters that determine the symmetric and asymmetric broadening of the relaxation peaks, respectively. The power law exponent $n$ would be 1 for pure dc conductivity, but due to polarization effects the best agreement with the experimental data was obtained for slightly lower values in the range 0.7-1, depending on the temperature and sample. It should also be noted that at a given temperature only two relaxation processes were normally present in the experimental window, as shown in Fig. 3 to give an example of how one of the spectra shown in Fig. 2(a) has been curve fitted. Thus, at higher temperatures most spectra were described by the conductivity term and two relaxation processes ( $\alpha$ and $w$ (or $\beta$ )) and at lower temperatures by only two relaxation processes $\left(w\right.$ (or $\beta$ ) and $w_{l}$ ). By using this curve fitting approach to describe the experimental data we were able to determine the temperature dependent relaxation times $\tau_{\alpha}$, $\tau_{w}$, and $\tau_{w l}$ of the $\alpha, w$ (or $\beta$ ), and $w_{l}$ relaxations, as shown in Fig. 4 for intercalated PG-water (a) and PGME-water (b) solutions.

The temperature dependences of the relaxation times will be discussed below, but before we do that we have to motivate our assignments of the dielectric loss peaks shown in Figs. 2 and 3. Interpretations of dielectric loss peaks are not obvious and therefore assignments can only be made by using all available knowledge about the typical behavior of a certain type of relaxation process, such as the viscosity related $\alpha$-relaxation or a more local $\beta$-relaxation. It is also valuable to compare the results with previous results for similar samples, particularly in the case of water containing samples since it has been shown ${ }^{22,23}$ that widely different water containing systems exhibit a nearly universal water relaxation at low temperatures. At least at higher water concentrations

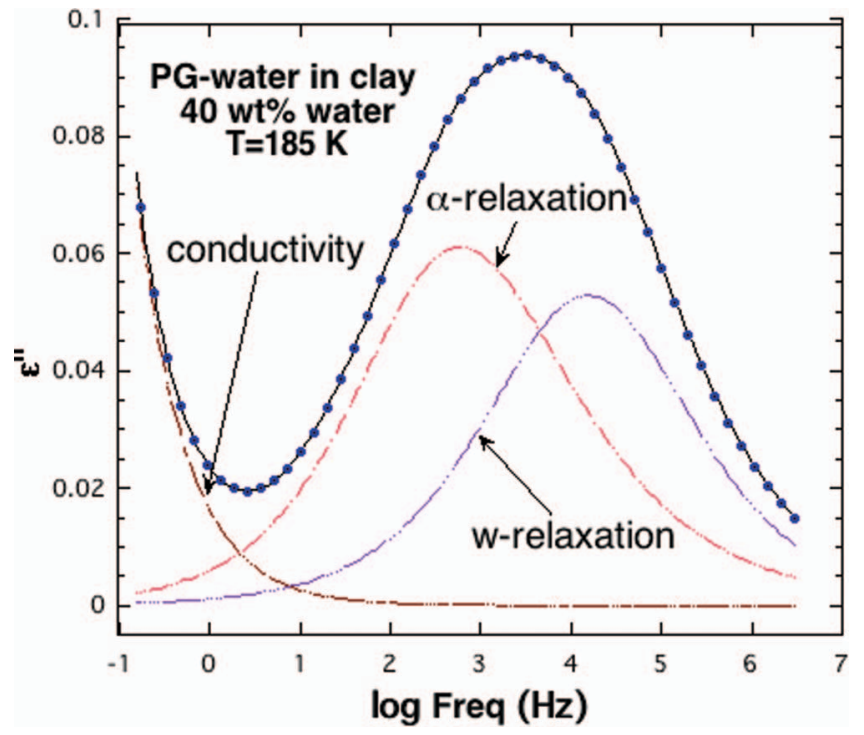

FIG. 3. A description of the fitting procedure of the data shown in Fig. 2 at $\mathrm{T}=185 \mathrm{~K}$. The solid line is the resulting fit to the experimental data, using Eq. (1). At this temperature the measured data are well described by a power law for the dc-conductivity, a Havriliak-Negami function ${ }^{19}$ for the $\alpha$-relaxation and a Cole-Cole function ${ }^{20}$ for the contribution from the main water $(w)$ relaxation and the $\beta$-relaxation of PG. Further details about the fitting procedure are provided in the main text.

where water clusters are formed both the time scale and activation energy of this water relaxation is basically the same for all systems. ${ }^{22,23}$ Thus, at higher water concentrations it should be easy to identify this water relaxation, although it has recently been found ${ }^{24}$ that also the $\beta$-relaxation of PG participates in this water relaxation. This implies that the local $\beta$-relaxation of PG becomes coupled to the water relaxation. For solutions of low water content, where more or less single water molecules are interacting with solute molecules, a previous study ${ }^{25}$ has indicated an opposite scenario with water molecules strongly affected by the $\beta$-relaxation of the solute molecules. Therefore, for the major part of the water concentration range we should expect to see a "combined" relaxation process, containing both the main $w$-relaxation of water and the $\beta$-relaxation of PG or PGME. Due to the high dipole moment of water the main contribution to this "combined" process should come from the water at high or intermediate water concentrations.

Further guidance in the interpretations is provided by the fact that the viscosity related $\alpha$-relaxation generally exhibits not only a different peak shape, i.e., asymmetric, than more local and symmetric secondary relaxation processes, such as the water relaxation and the $\beta$-relaxation of $\mathrm{PG}$, but also a different temperature dependence. The structural $\alpha$-relaxation exhibits a non-Arrhenius type temperature dependence that is commonly described by the Vogel-Fulcher-Tammann (VFT) function $^{26-28}$

$$
\tau_{\alpha}=\tau_{0} \exp \left(\frac{\mathrm{DT}_{0}}{\mathrm{~T}-\mathrm{T}_{0}}\right)
$$

where $\tau_{0}$ is the relaxation time extrapolated to infinite temperature and $T_{0}$ is the temperature where the relaxation time $\tau_{\alpha}$ extrapolates to infinity. The parameter $D$ determines the 



FIG. 4. Temperature dependences of the relaxation times obtained from the curve fitting procedure shown in Fig. 3 and described in the main text. Relaxation times for the intercalated solutions of PG-water and PGME-water are shown in (a) and (b), respectively. Relaxation times of the $\alpha$-relaxation are given by solid symbols, the $w$ (or $\beta$ ) relaxation by open symbols and the fast local water relaxation $w_{l}$ by different types of crosses. The VFT equation (Eq. (2)) was always used to describe the temperature dependence of $\tau_{\alpha}$, whereas the temperature dependence of $\tau_{w}$ (or $\tau_{\beta}$ ) was described by either the Arrhenius equation (Eq. (3)) or the VFT equation, depending on the temperature and the water concentration. The temperature dependence of $\tau_{w l}$ was always described by the Arrhenius equation. Temperature ranges (from onset to end) of the calorimetric $T_{g}$ are also shown for comparison with the dielectrically obtained dynamic glass transition temperatures, as obtained when $\tau_{\alpha}$ reaches $100 \mathrm{~s}$. The fit parameters used to describe the temperature dependences are provided in Table I for the VFT-fits and Table II for the Arrheniusfits.

deviation from Arrhenius temperature dependence (large $D$ value means a small deviation), and it is related to the fragility of the glass forming liquid. The $w$-relaxation and other more local relaxation processes ( $\beta$-relaxations) are decoupled from the structural $\alpha$-relaxation in the glassy and deeply super- cooled regimes, where their temperature dependences are given by the Arrhenius equation,

$$
\tau_{\beta}=\tau_{0} \exp \left(\frac{\mathrm{E}_{\mathrm{a}}}{\mathrm{k}_{\mathrm{B}} \mathrm{T}}\right)
$$

where $E_{a}$ is the activation energy of the relaxation process. However, at higher temperatures such $\beta$-relaxations tend to be merged with the $\alpha$-relaxation or at least follow a similar VFT behavior. The apparent non-Arrhenius temperature dependence of $\beta$-relaxations at higher temperatures can also be explained by Williams Ansatz, ${ }^{29,30}$ as a mathematical consequence of their merging with the $\alpha$-relaxation. Thus, it is typical that the apparent temperature dependence of the $w$ relaxation and other types of $\beta$-relaxations changes from a low temperature Arrhenius behavior to a high temperature VFT dependence. ${ }^{22}$ For supercooled liquids and aqueous solutions such dynamic crossovers are generally occurring at $T_{g}$ or slightly above.

With this knowledge about peak shapes and temperature dependences of different types of relaxation processes it is possible to assign the dielectric loss peaks shown in Figs. 2 and 3, and their associated temperature dependent relaxation times, shown in Fig. 4.

Let us start to discuss the relaxation times shown in Fig. 4(a) for some of the investigated PG-water solutions confined in the Na-vermiculite clay. The $\alpha$ and $w$ relaxations are both expected, as discussed above, and, in fact, also the fastest water relaxation, $w_{l}$, is commonly observed ${ }^{31}$ for deeply supercooled or glassy water in different types of confined geometries, such as the present Na-vermiculite clay. ${ }^{4}$ This local water relaxation has also been observed in a wide range of systems by quasielastic neutron scattering, ${ }^{32}$ but the physical nature of this low temperature process is still not fully clear and it will not be further discussed in this paper.

In Fig. 4(a) it can also be seen that the temperature dependence of both the $\alpha$-relaxation and the $w$-relaxation is well described by the VFT equation at temperatures above $T_{g}$ and water concentrations up to $40 \mathrm{wt}$. $\%$. At $100 \mathrm{wt}$. \% water no $\alpha$-relaxation is observed, as typical for confined supercooled water, ${ }^{5}$ and only an Arrhenius temperature dependent water relaxation can be observed, in agreement with previous dielectric relaxation studies of water intercalated in clay. ${ }^{4}$ However, at 80 wt. \% water an interesting phenomenon is observed. In this case the $\alpha$-relaxation can be observed, but at the same time the $w$-relaxation behaves as no $\alpha$-relaxation is present, i.e., it does not merge with the $\alpha$-relaxation or shows a dynamic crossover, but instead crosses the $\alpha$-relaxation about $10 \mathrm{~K}$ above $T_{g}$. This phenomenon can only occur if the two relaxation processes are independent of each other, which requires that they do not occur in the same parts of the confined solution. The most probable explanation for this behavior is that the interlayer $\mathrm{Na}^{+}$ions are hydrated by water molecules, and that it is this hydration shell which is responsible for this water relaxation. Apparently, these water molecules do not participate in the $\alpha$-relaxation. However, it is evident that some of the water molecules are also participating in the $\alpha$-relaxation, since this process exhibits a dynamic crossover just before it reaches a time scale of $100 \mathrm{~s}$. This time scale is commonly defined as a dielectric or 
dynamic glass transition temperature, and it is often in good agreement with the calorimetric $T_{g}$, as shown in Fig. 4(a). The observed crossover is in reality a decoupling of a water relaxation from the cooperative $\alpha$-relaxation. Thus, below $T_{g}$ two $w$-relaxations are observed, a faster one for the water molecules associated with the interlayer $\mathrm{Na}^{+}$ions and a slower one for water associated with the PG molecules. Here, it should also be noted that such a dynamic crossover, due to a decoupling of the water relaxation from the structural $\alpha$-relaxation, is basically always observed for aqueous bulk solutions where the $\beta$-relaxation of the solute molecules is weak compared to the $\alpha$-relaxation, as is the case for the corresponding bulk solutions. ${ }^{1}$ The decoupling occurs closer and closer to the $T_{\mathrm{g}}$-related time scale of $100 \mathrm{~s}$ with decreasing water content of the solution, because the water relaxation slows down with decreasing water concentration. By comparing with the behavior of the $w$-relaxation at $20 \mathrm{wt}$. \% water (where it cannot even be seen), 40 wt. \% water (where it is almost one order of magnitude faster), and the corresponding bulk solution with $30 \mathrm{wt}$. \% water (where it is almost identical ${ }^{1}$ ) we conclude that the effective water concentration in these PG-rich regions is likely to be around $30 \mathrm{wt}$. \%. Hence, a substantial amount of all the water seems to be associated with the interlayer $\mathrm{Na}^{+}$ions at a water concentration of $80 \mathrm{wt} . \%$. At lower water concentrations no such independently relaxing hydration shell is present, since no water relaxation is then decoupled from the $\alpha$-relaxation at temperatures above $T_{g}$.

Let us now turn to Fig. 4(b) and the intercalated PGMEwater solutions. Qualitatively, the relaxation behavior appears to be rather similar to the PG-water system. However, it can be seen that one VFT temperature dependent relaxation process is "missing." Furthermore, an extrapolation of the observed VFT temperature dependent process to a time scale of $100 \mathrm{~s}$, i.e., to its dynamic glass transition temperature, is only consistent with the calorimetric $T_{g}$ for the intercalated solutions containing 0 and 20 wt. \% water, as shown in the figure. For the samples of higher water contents the relaxation process is not as slow as expected from the calorimetrically obtained $T_{g}$-values. This fact suggests that the $\alpha$-relaxation is only observed for the two lowest water concentrations, whereas for the higher water concentrations the observed dielectric loss peak is mainly due to the $w$-relaxation, although its nonArrhenius temperature dependence at higher temperatures indicates that it is affected by the $\alpha$-relaxation, although this latter relaxation is too weak to be clearly observed. It should here be noted that geometrical confinements are known to reduce the intensity of the cooperative $\alpha$-relaxation considerably more than for more local $\beta$-relaxations. ${ }^{33}$ This is also evident in Fig. 5, which compares the intensities of the confined solutions containing $40 \mathrm{wt}$. \% water with the corresponding bulk solutions. The $\alpha$-relaxation dominates for the bulk solutions, whereas the more local $w$-relaxation is considerably stronger for the confined solutions. Thus, it is likely that the confinement has made the $\alpha$-relaxation so weak for the samples of higher water contents that it cannot be clearly observed. Instead, the dielectric loss data are dominated by the $w$-relaxation, which, in analogy to the corresponding PGwater solutions, exhibits a dynamic crossover to a low tem-



FIG. 5. Imaginary part of the dielectric permittivity of bulk and intercalated PG-water mixtures as well as bulk and intercalated PGME-water mixtures. For a direct comparison between the four systems the water content was about $40 \mathrm{wt}$. \% in each solution and the temperature approximately $170 \mathrm{~K}$.

perature Arrhenius dependence when it decouples from the (non-observable) $\alpha$-relaxation. Finally, it is clear that the same kind of hydration shell around the interlayer $\mathrm{Na}^{+}$ions, as discussed for the PG-water solutions, develops with increasing water content for the intercalated PGME-water solutions. This is indicated by a crossing of the "universal" low temperature water relaxation and the non-observable, but expected, $\alpha$ relaxation already at a water content of $60 \mathrm{wt}$. \% (not shown in Fig. 4(b) for clarity, but instead shown in Fig. 6(b)). Except for these differences and the different relaxation times of the processes in the two systems their relaxation behaviors are similar, as also indicated by the fit parameters used to describe the temperature dependences of the relaxation times, given in Table I for the VFT-fits and in Table II for the Arrhenius-fits. These tables show that there are no systematic differences between the fit parameters obtained for the different samples, i.e., no systematic difference between the confined solutions of PG and PGME and no systematic dependence on the water concentration. Only the parameters obtained for the low temperature Arrhenius-fit of the $w$ (or $\beta$ ) relaxation in the confined PGME solution containing 40 wt. $\%$ water are substantially different compared to the corresponding parameters for the other samples. However, in this case the Arrhenius-fit was based on only three data points, and the error bars are consequently large. Within the errors of the fitting procedure, the values of all parameters can be regarded as physically realistic.

Fig. 5 shows, as mentioned above, comparisons with the corresponding bulk data. Two major differences can be observed between the intercalated and bulk solutions. First, the intensities (or dielectric strengths) of the intercalated solutions are about two orders of magnitude lower than for the corresponding bulk solutions, and, second, the peak frequency of the dielectric loss has moved to a higher frequency for the 

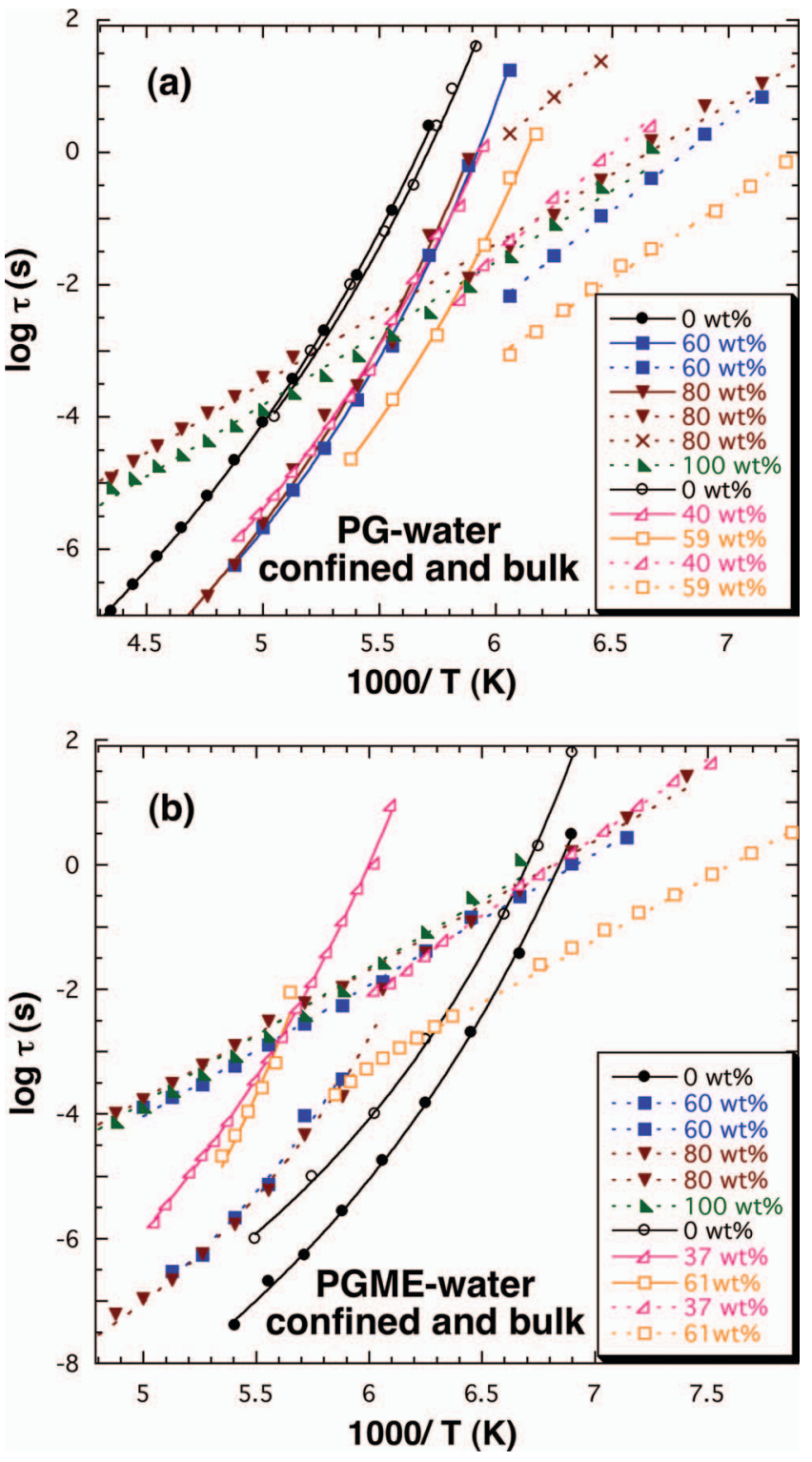

FIG. 6. Comparison of relaxation times of some confined (solid symbols) and bulk (open symbols) solutions. The temperature dependences of $\tau_{\alpha}$ are described by the VFT equation (solid lines), whereas the fits to $\tau_{w}$ (or $\tau_{\beta}$ ) (dashed lines) are made by either the VFT equation or the Arrhenius equation, depending on the sample and temperature. The water content of each solution is given in the figure. The PG-water systems are compared in (a) and the PGME-water systems are compared in (b). Bulk data have been taken from Ref. 1.

intercalated solutions. These differences are related and caused by the substantial decrease of the dielectric strength, particularly of the $\alpha$-relaxation, in the confined geometry. ${ }^{33}$ The shift of the peak frequency in the confinement is therefore mainly due to that the $w$-relaxation becomes stronger than the $\alpha$-relaxation, rather than that the dielectric loss peak of the $\alpha$ relaxation shifts to a considerably higher frequency, i.e., that the glass transition related $\alpha$-relaxation becomes faster. The almost preserved relaxation time of the $\alpha$-relaxation is further supported by the observation that the calorimetric $T_{g}$ is basically not affected by the confinement. In Figs. 6(a) and 6(b) comparisons between some of the confined and bulk solutions of PG-water and PGME-water, respectively, are shown for the
TABLE I. Fit parameters of all temperature dependences of relaxation times described by the VFT equation.

\begin{tabular}{lcccc}
\hline \hline Sample & Relaxation process & $\tau_{0}(\mathrm{~s})$ & $T_{0}(\mathrm{~K})$ & Fragility $D$ \\
\hline PG, 0 wt. \% water & $\alpha$ & -14.7 & 114 & 18.5 \\
PG, 0 wt. \% water & Combined $\beta \& w$ & -14.1 & 127 & 11.4 \\
PG, 20 wt. \% water & $\alpha$ & -14.8 & 114 & 17.5 \\
PG, 20 wt. \% water & Combined $\beta \& w$ & -13.7 & 124 & 10.8 \\
PG, 40 wt. \% water & $\alpha$ & -13.2 & 124 & 11.2 \\
PG, 40 wt. \% water & Combined $\beta \& w$ & -13.1 & 132 & 6.9 \\
PG, 60 wt. \% water & $\alpha$ & -13.5 & 123 & 11.9 \\
PG, 60 wt. \% water & Combined $\beta \& w$ & -13.5 & 119 & 10.7 \\
PG, 80 wt. \% water & $\alpha$ & -14.7 & 119 & 14.8 \\
PGME, 0 wt. \% water & $\alpha$ & -14.5 & 107 & 12.1 \\
PGME, 20 wt. \% water & $\alpha$ & -16.1 & 104 & 17.5 \\
PGME, 40 wt. \% water & Combined $\beta \& w$ & -15.4 & 98 & 19.6 \\
PGME, 60 wt. \% water & Combined $\beta \& w$ & -14 & 114 & 12.1 \\
PGME, 80 wt. \% water & Combined $\beta \& w$ & -12.2 & 126 & 7 \\
\hline
\end{tabular}

relaxation times of $\alpha$ and $w$ (or $\beta$ ). It should here be noted that in the case of the PGME-water system a proper comparison of the $\alpha$-relaxation time can only be made for PGME without water, since the $\alpha$-relaxation of the confined solution is only visible up to 20 wt. \% water, as shown in Fig. 4(b). Hence, at the higher water concentrations we have to assume that the $\alpha$-relaxation time is only slightly affected by the confinement due to the small difference in the calorimetric $T_{g}$.

Figure 6 shows also that the relaxation time of $w$ is in general weakly influenced by the restricted 2-dimensional geometry. Only at the highest possible water concentration (before crystallization to ice occurs) of about $60 \mathrm{wt}$ \% in the bulk solutions the $w$-relaxation is substantially faster than in the confinement. We believe the reason for this is that the water relaxation in the clay is slowed down by the interaction with the intercalated $\mathrm{Na}^{+}$ions, and this interaction is more dominating at high water concentrations. At least at higher temperatures, such slowing down of the water dynamics has been established by neutron scattering. ${ }^{14}$ This also leads to a

TABLE II. Fit parameters of all temperature dependences of relaxation times described by the Arrhenius equation.

\begin{tabular}{lclc}
\hline \hline Sample & Relaxation process & $\tau_{0}(\mathrm{~s})$ & $E_{a}(\mathrm{~kJ} / \mathrm{mol})$ \\
\hline PG, 40 wt. \% water & Combined $\beta \& w$ & -15.9 & 50 \\
PG, 40 wt. \% water & $w_{l}$ & -17.5 & 46 \\
PG, 60 wt. \% water & $w$ & -19 & 54 \\
PG, 60 wt. \% water & Combined $\beta \& w$ & -14 & 42 \\
PG, 80 wt. \% water & Water & -16.6 & 54 \\
PG, 80 wt. \% water & Combined $\beta \& w$ & -14.6 & 42 \\
PGME, 40 wt. \% water & Combined $\beta \& w$ & -23 & 68 \\
PGME, 60 wt. \% water & $w$ & -14.5 & 42 \\
PGME, 60 wt. \% water & $w_{l}$ & -17.8 & 48 \\
PGME, 80 wt. \% water & $w$ & -14 & 42 \\
PGME, 80 wt. \% water & $w_{l}$ & -18 & 45 \\
PGME, 80 wt. \% water & Combined $\beta \& w$ & -12 & 38 \\
100 wt. \% water & $w$ & -14.6 & 42 \\
100 wt. \% water & $w_{l}$ & -18.8 & 54 \\
\hline \hline
\end{tabular}


less pronounced speeding up of the $w$-relaxation with increasing water content in the confinement.

\section{CONCLUSIONS}

The present dielectric relaxation study of PG-water and PGME-water solutions confined in a Na-vermiculite clay can be regarded as consistent with previous studies ${ }^{33,34}$ of the same solutes confined in the same type of clay. As in these previous studies, we find that the time scale of the glass transition related dynamics is only weakly altered compared to bulk, despite the very severe geometrical restriction to onetwo molecular layers in the direction perpendicular to the clay platelets. In the case of the confined PG-water solutions it is an expected finding, due to the small confinement effect previously observed for PG and the weak effect of added water to the corresponding bulk system. However, for the PGMEwater system it is remarkable that the concentration dependence of the glass transition temperature shows the same non-monotonic behavior as in the corresponding bulk system, indicating that the same type of large hydrogen bonded relaxing entities, with water bridging between different PGME molecules, are formed. This further suggests that a normally three-dimensional hydrogen bonded network has the ability to adapt to a two-dimensional geometry. Another interesting observation is that the local water relaxation does not merge with the structural $\alpha$-relaxation (or the $\beta$-relaxation of PGME in the case when $\alpha$ cannot be directly observed) at higher water contents. This implies that water domains, with the same relaxation processes as in fully hydrated clay, must be formed at these water concentrations. Most likely, the tendency of the interlayer $\mathrm{Na}^{+}$ions to hydrate gives rise to a water hydration shell around each $\mathrm{Na}^{+}$ion. On the other hand, at lower water contents the water relaxation seems to be coupled to the $\beta$ relaxation of the solute molecules, since only one combined relaxation is observed for these two processes. Finally, it can be concluded that the interaction to the clay platelets does not seem to have any substantial effect on the hydrogen bonds between the molecules in the solutions. Except for the formation of hydration shells around the interlayer $\mathrm{Na}^{+}$ions at high water contents, the results suggest that the amount and character of the hydrogen bonds are bulk-like.

\section{ACKNOWLEDGMENTS}

This work was financially supported by the Swedish Research Council and the Swedish Energy Agency.
${ }^{1}$ J. Sjöström, J. Mattsson, R. Bergman, E. Johansson, K. Josefsson, D. Svantesson, and J. Swenson, Phys. Chem. Chem. Phys. 12, 10452 (2010).

${ }^{2}$ J. Sjöström, J. Mattsson, R. Bergman, and J. Swenson, Phys. Chem. B 115, 10013 (2011).

${ }^{3}$ J. Swenson, K. Elamin, H. Jansson, and S. Kittaka, Chem. Phys. 424, 20 (2013).

${ }^{4}$ R. Bergman and J. Swenson, Nature 403, 283 (2000).

${ }^{5}$ K. Elamin, H. Jansson, S. Kittaka, and J. Swenson, Phys. Chem. Chem. Phys. 15, 18437 (2013).

${ }^{6}$ A. Nagoe and M. Oguni, J. Phys.: Condens. Matter 25, 465110 (2013).

${ }^{7}$ C. E. Bertrand, K.-H. Liu, E. Mamontov, and S.-H. Chen, Phys. Rev. E 87, 042312 (2013)

${ }^{8}$ A. Lerbret, G. Lelong, P. E. Mason, M. L. Saboungi, and J. W. Brady, J. Phys. Chem. B 115, 910 (2011).

${ }^{9}$ N. Choudhury, J. Chem. Phys. 132, 064505 (2010).

${ }^{10}$ M. Sattig and M. Vogel, J. Chem. Phys. Lett. 5, 174 (2014).

${ }^{11}$ E. Mamontov, D. R. Cole, S. Dai, M. D. Pawel, C. D. Liang, T. Jenkins, G. Gasparovic, and E. Kintzel, Chem. Phys. 352, 117 (2008).

${ }^{12}$ M. Oguni, Y. Kanke, A. Nagoe, and S. Namba, J. Phys. Chem. B 115, 14023 (2011).

${ }^{13}$ J. Sjöström, J. Swenson, R. Bergman, and S. Kittaka, J. Chem. Phys. 128, 154503 (2008).

${ }^{14}$ J. Swenson, R. Bergman, and S. Longeville, J. Chem. Phys. 115, 11299 (2001).

${ }^{15}$ L. M. Anovitz, E. Mamontov, P. Ben Ishai, and A. I. Kolesnikov, Phys. Rev. E 88, 053206 (2013).

${ }^{16}$ S. Beckert, M. Gratz, J. Kullmann, D. Enke, and F. Stallmach, Appl. Magn. Reson. 44, 827 (2013).

${ }^{17}$ G. Lelong, D. L. Price, J. W. Brady, and M. L. Saboungi, J. Chem. Phys. 127, 065102 (2007).

${ }^{18}$ J. Sjöström, R. Bergman, J. Swenson, C. Wadell, T. Moberg, and J. Mattsson, Phys. Chem. B 115, 1842 (2011).

${ }^{19}$ R. Richert, A. Agapov, and A. P. Sokolov, J. Chem. Phys. 134, 104508 (2011).

${ }^{20}$ S. Havriliak and S. Negami, Polymer 8, 161 (1967).

${ }^{21}$ K. S. Cole and R. H. Cole, J. Chem. Phys. 9, 341 (1941).

${ }^{22}$ S. Cerveny, G. A. Schwartz, R. Bergman, and J. Swenson, Phys. Rev. Lett. 93, 245702 (2004).

${ }^{23}$ S. Cerveny, A. Alegria, and J. Colmenero, Phys. Rev. E 77, 031803 (2008).

${ }^{24}$ D. Sauer, B. Schuster, M. Rosenstihl, S. Schneider, V. Talluto, T. Walther, T. Blochowicz, B. Stühn, and M. Vogel, J. Chem. Phys. 140, 114503 (2014).

${ }^{25}$ K. Elamin, J. Sjöström, H. Jansson, and J. Swenson, J. Chem. Phys. 136, 104508 (2012).

${ }^{26}$ H. Vogel, Phys. Z. 22, 645 (1921).

${ }^{27}$ G. S. Fulcher, J. Am. Ceram. Soc. 8, 339 (1925).

${ }^{28}$ G. Tammann, W. Hesse, and Z. Anorg, Allg. Chem. 156, 245 (1926).

${ }^{29}$ G. Williams, Adv. Polym. Sci. 33, 60 (1979).

${ }^{30}$ R. Bergman, F. Alvarez, A. Alegria, and J. Colmenero, J. Chem. Phys. 109, 7546 (1998)

${ }^{31}$ J. Swenson, H. Jansson, J. Hedström, and R. Bergman, J. Phys.: Condens. Matter 19, 205109 (2007).

${ }^{32}$ F. Mallamace, C. Corsaro, P. Baglioni, E. Fratini, and S.-H. Chen, J. Phys.: Condens. Matter 24, 064103 (2012).

${ }^{33}$ R. Bergman, J. Mattsson, C. Svanberg, G. A. Schwartz, and J. Swenson, Europhys. Lett. 64, 675 (2003).

${ }^{34}$ S. Cerveny, J. Mattsson, J. Swenson, and R. Bergman, J. Phys. Chem. B 108, 11596 (2004). 